\title{
Análise da Revisão Cochrane: Anticoagulação versus Placebo em Doentes com Insuficiência Cardíaca em Ritmo Sinusal. Cochrane Database Syst Rev. 2014;3:CD003336.
}

\author{
Analysis of the Cochrane Review: Anticoagulation versus Placebo for Heart \\ Failure in Sinus Rhythm. Cochrane Database Syst Rev. 2014;3:CD003336.
}

\author{
Daniel CALDEIRA 1,2,3, António VAZ-CARNEIRO ${ }^{4,5}$, João COSTA ${ }^{1,2,4,5}$ \\ Acta Med Port 2014 May-Jun;27(3):284-286
}

\section{RESUMO}

Os eventos trombóticos e embólicos contribuem para a morbilidade e mortalidade associada à Insuficiência Cardíaca Crónica (IC). Diferentemente da associação da fibrilhação auricular (FA) com IC, em que o benefício da anticoagulação está bem documentada, a utilização desta classe farmacoterapêutica naqueles que se apresentam em ritmo sinusal (sem história pregressa de FA) é discutível. Nesta revisão sistemática da Cochrane Collaboration, os autores avaliaram os benefícios e riscos associados à anticoagulação oral (versus placebo) nesta população. Apenas foram publicados 2 ensaios clínicos aleatorizados e controlados (um dos quais com desenho aberto) incluindo um total de 324 doentes. Os resultados da meta-análise baseados na melhor evidência disponível não suporta o uso sistemático de anticoagulantes orais em doentes com IC e ritmo sinusal na prevenção de morte (global ou cardiovascular) ou eventos cardiovasculares não-fatais. A anticoagulação aumentou significativamente o risco de hemorragia grave.

Palavras-chave: Anticoagulação; Insuficiência Cardíaca; Ritmo Sinusal; Revisão Sistemática.

\section{ABSTRACT}

Thrombotic and embolic events contribute to the morbidity and mortality associated to Chronic Heart Failure (HF). Differently from patients with atrial fibrillation (AF) and HF, in which the benefit of anticoagulation is well documented, the use of these drugs in those with HF in sinus rhythm (without AF history) is controversial. In this systematic review from the Cochrane Collaboration, the authors evaluated the benefits and risks associated with oral anticoagulation (versus placebo) in this population. Only 2 randomized controlled trials were published (one with open-label design) enrolling a total of 324 patients. The results of the meta-analysis based on the best available evidence do not support the systematic use of oral anticoagulants in patients with HF and sinus rhythm for preventing death (overall or cardiovascular) or non-fatal cardiovascular events. Furthermore the major bleeding risk was significantly increased.

Keywords: Anticoagulation; Heart Failure; Sinus Rhythm; Systematic Review.

\section{QUESTÃO CLÍNICA}

A anticoagulação reduz o risco de eventos cardiovasculares em doente com insuficiência cardíaca em ritmo sinusal?

\section{OBJECTIVOS}

Esta revisão sistemática procurou avaliar, em doentes com Insuficiência Cardíaca (IC) crónica em ritmo sinusal e sem história pregressa de fibrilhação auricular (FA), o benefício clínico da anticoagulação oral de longa duração nos seguintes outcomes de eficácia:

1. Mortalidade global

2. Mortalidade cardiovascular

3. Eventos tromboembólicos

\section{METODOLOGIA}

Revisão sistemática de ensaios clínicos aleatorizados (RCTs) que incluiram doentes com IC crónica em ritmo sinusal alocados a terapêutica anticoagulante ou placebo. ${ }^{1}$ Fo- ram também incluídos ensaios quasirandomized (QRCTs), que são ensaios cujo método de aleatorização não garante a ocultação ou imprevisibilidade da mesma (por exemplo, data de nascimento, número de registo hospitalar, etc.), e portanto, estão mais sujeitos a vieses nomeadamente de selecção que os RCTs.

\section{RESULTADOS}

No total foram incluídos para análise cinco ensaios: dois RCTs (WASH e HELAS) com 324 doentes, ${ }^{2.3}$ e três QRCTs com 942 doentes.

A idade média foi de 59,9 anos (variação 20-89 anos), os RCTs envolveram predominantemente países europeus, enquanto os QRCTs foram realizados nos Estados Unidos da América.

Os QRCTs foram publicados entre 1950-1960 e sugeriram um potencial efeito benéfico da anticoagulação nos doentes com IC e ritmo sinusal. ${ }^{1}$

1. Laboratório de Farmacologia Clínica e Terapêutica. Faculdade de Medicina. Universidade de Lisboa. Lisboa. Portugal.

2. Unidade de Farmacologia Clínica. Instituto de Medicina Molecular. Faculdade de Medicina. Universidade de Lisboa. Lisboa. Portugal.

3. Serviço de Cardiologia. Hospital Garcia de Orta. Almada. Portugal.

4. Centro de Estudos de Medicina Baseada na Evidência. Faculdade de Medicina. Universidade de Lisboa. Lisboa. Portugal.

5. Centro Colaborador Português da Rede Cochrane Iberoamericana. Lisboa. Portugal.

Recebido: 05 de Junho de 2014 - Aceite: 09 de Junho de 2014 | Copyright $\odot$ Ordem dos Médicos 2014 


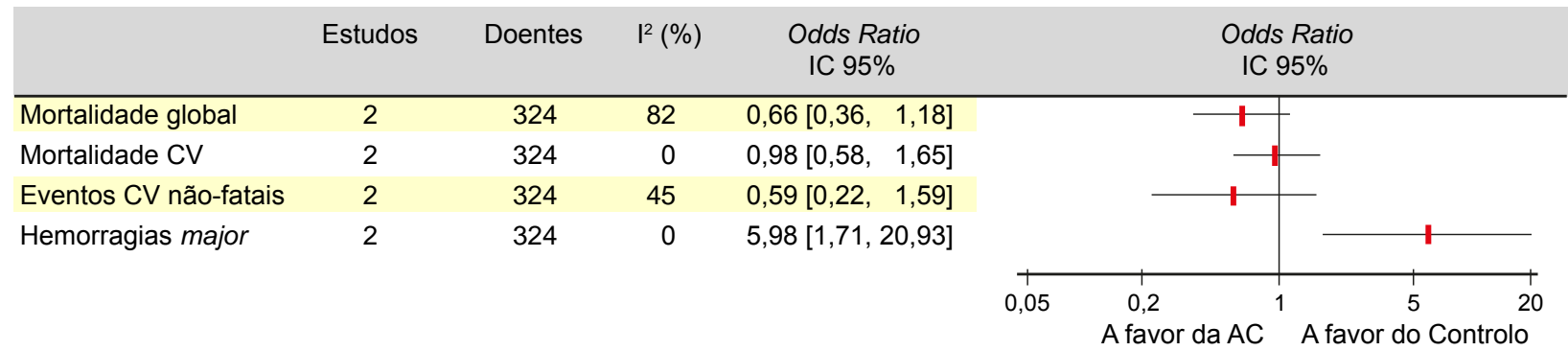

Figura 1 - Resultados da meta-análise. Adaptado de Lip et al. ${ }^{1}$

AC: Anticoagulação; CV: Cardiovascular; ${ }^{2}$ : percentagem de variabilidade na estimativa final devida a heterogeneidade entre os estudos incluídos.

Em relação aos RCTs, o estudo WASH, apresentava um desenho aberto, mas com a avaliação dos outcomes em ocultação. Este RCT incluiu 254 doentes com IC $(60 \%$ de etiologia isquémica) em ritmo sinusal: 80 doentes tratados com varfarina (INR alvo 2,5), 80 doentes tratados com ácido acetilsalicílico, e 94 doentes sem terapêutica anti-trombótica. ${ }^{2}$ Para a revisão foram incluidos os doentes tratados com varfarina e aqueles sem terapêutica anti-trombótica. O período médio de seguimento foi 2,3 anos. ${ }^{2} \mathrm{O}$ outro RCT incluido foi o HELAS, ${ }^{3}$ com teve duas comparações: varfarina versus ácido acetilsalicílico em doentes com IC de etiologia isquémica (não incluída nesta revisão devido à ausência de grupo controlo com placebo); e varfarina (INR alvo 2-3) versus placebo em 82 doentes com IC de etiologia não-isquémica, dilatação ventricular, em ritmo sinusal. ${ }^{3} \mathrm{O}$ seguimento médio do estudo foi de 1,8 anos. ${ }^{3}$

Em ambos RCTs, o outcome primário foi um outcome composto: primeira ocorrência de morte ou de eventos cardiovasculares não-fatais. Não se constataram diferenças significativas entre as intervenções (anticoagulaçãoversus placebo) no que diz respeito aos resultados primários dos RCTs. ${ }^{2,3}$ A meta-análise dos resultados agregados destes ensaios (Fig. 1) também não mostra um benefício da anticoagulação. ${ }^{1} \mathrm{O}$ único resultado significativo foi o aumento do risco de hemorragia major com a anticoagulação (Fig. 1). ${ }^{1}$

\section{CONCLUSÕES}

Apesar da anticoagulação estar indicada em doentes com IC com outras indicações para esta terapêutica (por exemplo, fibrilhação auricular), a presente revisão mostra que a melhor evidência disponível não suporta o uso da anticoagulação para modificação do prognóstico em doentes com IC que permanecem em ritmo sinusal.

\section{COMENTÁRIO}

A IC é uma síndrome cada vez mais prevalente com morbidade e mortalidade significativas. ${ }^{4}$ Nas próximas dé- cadas perspectiva-se um aumento dos custos associados, dado o aumento de doenças associadas e fatores de risco relacionados com a IC, bem como pelo envelhecimento da população. ${ }^{5,6}$ Estudos observacionais sugeriram uma associação positiva entre IC crónica, alterações na hemostase e eventos tromboembólicos ${ }^{7,8} \mathrm{Em}$ doentes com fibrilhação auricular, a existência de IC ou disfunção ventricular esquerda aumenta o risco de tromboembolismo, e a anticoagulação oral diminui significativamente o risco de complicações tromboembólicas. ${ }^{9}$

Em relação aos doentes com IC que se mantêm em ritmo sinusal (sem história de fibrilhação auricular), a meIhor evidência disponível, agregada nesta revisão, é pouco robusta pelo número de estudos (2 RCTs), dimensão da amostra (324 doentes) e também pelo facto de um dos RCTs não ter sido realizado em ocultação. Contudo, os resultado individuais ou agregados destes estudos não suportam o uso de anticoagulação nestes doentes. ${ }^{1} \mathrm{O}$ grupo de trabalho de trombose da Sociedade Europeia de Cardiologia (que conta com o autor desta revisão sistemática, GY Lip), publicou um documento de consenso, não recomendando o uso de fármacos anti-trombóticos (em que se incluem anticoagulantes e antiagregantes) em doentes com insuficiência cardíaca sistólica e ritmo sinusal, ${ }^{10}$ na ausência de indicações formais concomitantes para anticoagulação (por exemplo, fibrilhação auricular) ou anti-agregação plaquetária (na doença coronária documentada, por exemplo). ${ }^{10}$

\section{IMPLICAÇÕES PARA PRÁTICA}

- Com base na melhor evidência disponível, não é possível concluir pela existência de um balanço benefício-risco positivo da anticoagulação oral em doentes com IC crónica que permanecem em ritmo sinusal e que não têm história pregressa de FA.

- Neste contexto, não se recomenda a sua utilização de rotina nestes doentes.

\section{REFERÊNCIAS}

1. Lip GY, Shantsila E. Anticoagulation versus placebo for heart failure in sinus rhythm. Cochrane Database Syst Rev. 201428;3:CD003336.

2. Cleland JG, Findlay I, Jafri S, Sutton G, Falk R, Bulpitt C, et al. The Warfarin/Aspirin Study in Heart failure (WASH): a randomized trial comparing antithrombotic strategies for patients with heart failure. Am
Heart J. 2004;148:157-64.

3. Cokkinos DV, Haralabopoulos GC, Kostis JB, Toutouzas PK, HELAS investigators. Efficacy of antithrombotic therapy in chronic heart failure: the HELAS study. Eur J Heart Fail. 2006;8:428-32.

4. Mosterd A, Hoes AW. Clinical epidemiology of heart failure. Heart. 
2007;93:1137-46.

5. Xuan J, Duong PT, Russo PA, Lacey MJ, Wong B. The economic burden of congestive heart failure in a managed care population. Am $J$ Manag Care. 2000;6:693-700.

6. Braunschweig F, Cowie MR, Auricchio A. What are the costs of heart failure? Europace. 2011;13(Suppl 2):ii13-7.

7. Hays AG, Sacco RL, Rundek T, Sciacca RR, Jin Z, Liu R, et al. Left ventricular systolic dysfunction and the risk of ischemic stroke in a multiethnic population. Stroke. 2006;37:1715-9.

8. Loh E, Sutton MS, Wun CC, Rouleau JL, Flaker GC, Gottlieb SS, et al. Ventricular dysfunction and the risk of stroke after myocardial infarction. N Engl J Med. 1997;336:251-7.
9. Camm AJ, Lip GY, De Caterina R, Savelieva I, Atar D, Hohnloser SH, et al. 2012 focused update of the ESC Guidelines for the management of atrial fibrillation: an update of the 2010 ESC Guidelines for the management of atrial fibrillation--developed with the special contribution of the European Heart Rhythm Association. Europace. 2012;14:1385413.

10. Lip GY, Ponikowski P, Andreotti F, Anker SD, Filippatos G, Homma S, et al. Thrombo-embolism and antithrombotic therapy for heart failure in sinus rhythm. A joint consensus document from the ESC Heart Failure Association and the ESC Working Group on Thrombosis. Eur J Heart Fail. 2012;14:681-95.

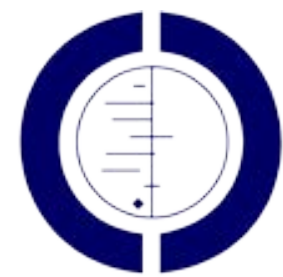

THE COCHRANE COLLABORATION 


\section{Análise da Revisão Cochrane: Anticoagulação versus Placebo em Doentes com Insuficiência Cardíaca em Ritmo Sinusal. Cochrane Database Syst Rev. 2014;3:CD003336. Acta Med Port 2014:27:284-286}

Publicado pela Acta Médica Portuguesa, a Revista Científica da Ordem dos Médicos

Av. Almirante Gago Coutinho, 151 1749-084 Lisboa, Portugal.

Tel: +351218428 215

E-mail: submissao@actamedicaportuguesa.com

www.actamedicaportuguesa.com ISSN:0870-399X | e-ISSN: 1646-0758

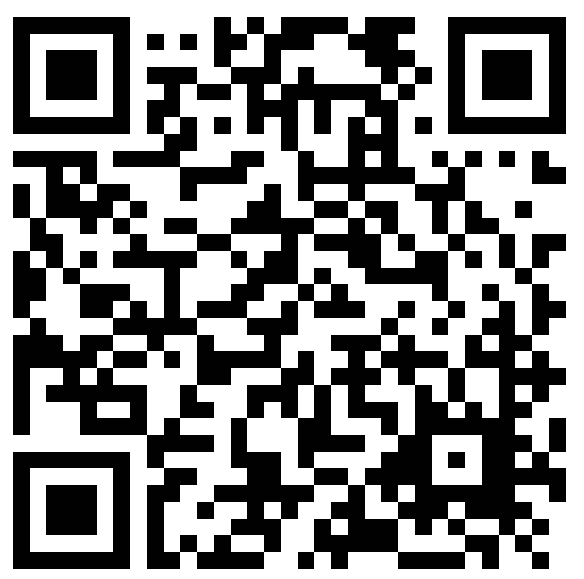

